\title{
Assessment of Regional Longitudinal Stain by Using Speckle Tracking Echocardiography - A Validation Study
}

\author{
Vitek Nili1 ${ }^{1}$, Bachner-Hinenzon Noa ${ }^{1}$, Lempel Meytal ${ }^{1}$, \\ Friedman Zvi ${ }^{2}$, Reisner Shimon ${ }^{3}$, Beyar Rafael ${ }^{1}$ and Adam Dan ${ }^{1}$ \\ ${ }^{1}$ The Faculty of Biomedical Engineering, \\ Technion-Israel Institute of Technology, Haifa, \\ ${ }^{2}$ General Electric Healthcare, \\ ${ }^{3}$ Department of Cardiology, Rambam Medical Center, \\ Faculty of Medicine, Technion-Israel Institute of Technology, Haifa
}

Israel

\section{Introduction}

Recently, there is an increasing body of evidence supporting the clinical importance of assessment of regional myocardial function as an essential tool for the evaluation of heart disease (Helm et al. 2005; Voigt et al. 2004, Liel-Cohen et al. 2010). In particular, numerous reports support the notion that diagnosis and management of coronary heart disease require the ability to adequately assess regional changes in myocardial function (Zwanenburg et al. 2005). Typical examples are the detection of ischemia or post acute myocardial infarction (AMI) during a stress test, even for nonocclusive coronary stenosis (Yip et al. 2004); Voigt and Flachskampf 2004), the differentiation of non-transmural vs. transmural infarct infarction, or of a reversible from non-reversible ischemic functional impairment.

One of the techniques currently in use for functional assessment of the cardiac muscle is Doppler based analysis of motion and deformation (Desco et al. 2002; Herbots et al. 2004; Voigt and Flachskampf 2004; Yip et al. 2003). The Doppler based techniques suffer from a number of critical limitations, the most significant one is that they are one dimensional and angle-dependent, leading to motion underestimation when the motion is not in the same orientation as the ultrasound beam at the point of measurement. Also, due to the cardiac movement during contraction, the segment being measured by the stable, but one dimensional beam, may be different at each moment. This limitation is significant since cardiac motion is very complex - at each location it consists of components that may either be independent from each other, such as heart translation, rotation and the active myocardial contraction, or that are physiologically related such as shortening along the myocardial fiber axis while thickening perpendicular to the fiber axis. These disadvantages limit the measurement to some sections of the heart, e.g. the septum and the free wall.

Ultrasound speckle tracking has been suggested for tissue strain and motion measurements (Bertrand et al., 1989; O'Donnell et al., 1991; O'Donnell et al., 1994). Recently, a different 
approach was suggested (Rappaport et al. 2006), also based on tracking of speckles (reflectors) in B-mode grayscale image sequences, which was reliable and efficient enough to be implemented in commercial systems. It suggested using an Acoustic Tagging method, which defines specific points that are being tracked over the time and finally the velocity in each Tracking Point (TP) is calculated (Behar et al. 2004). Acoustic Tagging is mimicking the idea used in tagged MRI, and the application of tagging for strain measurements. The implementation (AFI, GE Healthcare, Inc.) in the clinical setting allows computing motion and contraction information within the whole myocardial cross-section, directly from echocardiographic B-mode sequences. It provided in numerous clinical studies efficient diagnosis of myocardial dysfunction (De Boeck et al. 2006; Leitman et al. 2004; Reisner et al. 2004; Suffoletto et al. 2006; Yip et al. 2004; Zwanenburg et al. 2005).

The AFI tool is based on automatic initial selection (within the myocardial wall) of predominant features according to their brightness, size and persistence, and tracking them from one frame to the next. The changes in a feature position allow determining its velocity. The velocity field of these features is very noisy, thus at the center of each image section, e.g. of 50x50 pixels, a ' $\mathrm{TP}^{\prime}$ ' is defined, with velocity value of the average movement of that section. The TP's are aligned within a grid along the myocardium, to finally form 4 lines of points. The velocities of these points are filtered again, to allow calculation of the longitudinal Strain Rate (SR) by the derivative of longitudinal velocity component along the line of points, and the transverse SR by the derivative of transverse velocity component perpendicular to the line of points. Strain is calculated by time-integration of the SR. The 'AFI' averages the velocity field of the 4 lines of $\mathrm{TP}^{\prime}$ s into one line of 'knots' along the longitudinal orientation of the wall, thus variations between myocardial layers cannot be detected. It also employs robust smoothing, thus the spatial resolution is relatively low.

The present study was designed to replace the exceedingly smoothing method (performed on the results of the tracking) used by the 'AFI', by a more elaborate smoothing scheme that would reveal motion information of the different myocardial layers. This would allow obtaining 2D Strain and SR tensors at multiple points along and across the myocardial wall, allowing analysis and observation of local deformations (orientations and magnitudes).

The smoothing scheme used here is based on the wavelet shrinkage method, which is efficient in noise rejection by cancelling certain components, as a result of thresholding in the wavelet domain (Donoho 1995; Donoho and Johnstone 1994; Mallat 1998; Unser 1996). The threshold must be selected so that noisy and unrealistic movements/velocities are cancelled, while changes of the Strain and SR over time, along and across the myocardial wall, could be analyzed. The wavelet shrinkage denoising algorithm used here follows a method suggested to suppress Gaussian noise (Donoho and Johnstone 1994), which selects the thresholds depending on the variance of the noise components of the signal. The efficiency of this denoising algorithm relies on the choice of the wavelet basis, the estimation of noise level, the method of threshold selection and parameters specific to the application.

\section{Methods}

The raw data for this study comprised of the regional velocities, estimated from the B-mode cine loops by the speckle tracking algorithm, before application of any smoothing or filtration. The raw data may be viewed as a velocity function, sampled at 4 transmural 
locations across ("transverse" - T) the myocardial wall, and at 50-58 locations along ("longitudinal" - L) the LV cross-section, from base to apex and back to the other base along the myocardium ( $\left.\mathrm{TP}^{\prime} \mathrm{s}\right)$, and along one cardiac cycle. The ultrasound imaging system performs at frame rates of $60-90 \mathrm{fps}$, thus the number of samples along time is 60 to 90 accordingly.

The $\mathrm{L}$ and $\mathrm{T}$ components of the velocity are smoothed separately, by using a transformation from the Cartesian coordinate system to an (L,T) coordinate system, as follows: For each transversal line of four points - the inward direction is defined as the $\mathrm{T}$ axis, and the orthogonal direction is defined as the $\mathrm{L}$ axis.

\subsection{De-noising using wavelet functions}

The wavelet shrinkage method was applied to the myocardial velocities as described by Bachner-Hinenzon et al. (2010). At first, low tracking quality data points are replaced by a weighted average of their neighboring data points. Thereafter, an interpolation along the transmural orientation is performed. Subsequently, 3D wavelet de-noising is applied to the interpolated data. Following the wavelet decomposition, the Detail coefficients are thresholded by hard and soft threshold. For the first level, the Detail coefficients are removed completely (hard threshold) and for the second and third levels a soft universal threshold was used (Bachner-Hinenzon et al. 2010). Finally, to obtain the smoothed signal, the inverse of 3D discrete wavelet transform is computed using reconstruction filters in an iterated process.

The effectiveness of the wavelet shrinkage method was compared with a second smoothing method - a polynomial curve fitting method, which for the 3D case required 22 coefficients that were empirically chosen to estimate a polynomial of order 2 in space and of order 4 in time.

\subsection{Strain and SR calculation}

The tracking algorithm causes artifacts when the image quality is low, and even after the wavelet shrinkage smoothing operation, additional smoothing operation must be performed before regional Strain and SR are calculated. Strain is calculated at the center of each segment containing $12 \mathrm{TP}^{\prime} \mathrm{s}-6$ points along the L orientation, and 2 along the $\mathrm{T}$ orientation ( 2 adjacent layers across the LV wall). First, the center of such segment is calculated as a weighted moving average. Then, the location of each center in the next frame is found from the weighted average of the point's velocity.

Let $\mathrm{Rn}$ be the distance between two adjacent segment centers on the longitudinal axis at the $\mathrm{n}$ 'th frame, and $\mathrm{Rn}+1$ be the distance between the same two centers at the next frame. The relative shortening of the segment occurring between these two frames is:

$$
d S L(n)=\frac{R_{n+1}-R_{n}}{R_{n}}
$$

The accumulative strain of the segment at the $n$ 'th frame, relative to its initial length is:

$$
S L(n)=S L(n-1)+d S L(n)(1+S L(n-1))
$$

The SR is calculated as the time derivative of the Strain. 


\subsection{Evaluation of the methods using simulated data}

Sequences of velocity data have been generated by a software program, which modeled different movements, in order to accurately analyze the influence of different parameters of the algorithm and to allow quantification of the accuracy of measurement of smoothed motions. The simulated data also allowed accurate and reliable comparison between the wavelet shrinkage method and a second smoothing method - polynomial curve fitting method.

\subsubsection{Simulation of normal cardiac motion}

The complex myocardial motion was modeled by construction of a simplified heart motion model. A set of data points, taken from a clinical data set at end-diastole, served as the initial conditions (location of points) for the simulated contraction (Fig. 1(a)). To this set of data points, an analytic motion model was applied, as described in Fig. 1(b). The model describes the displacement of each point in the transmural and longitudinal direction, between 2 frames (i.e. 2 time samples), with delays among the 4 layers. It also includes a time dependant component common to all points, which describes the contraction and relaxations phases. By differentiating the displacement curves, the synthetic velocities in each layer are produced. The derived velocities (in each layer) are obtained in the transmural and longitudinal directions (see below). In order to test the algorithm under more realistic conditions, a random Gaussian noise was added (Noise $\sim N(0, \sigma), \sigma=10 \%$ of maximal velocity in each direction) to the velocities values. The quality of the results of each smoothing method were quantified in terms of the sum of square errors (SSE) between the denoised velocities (i.e. after applying the smoothing algorithm) and the original simulated velocities (before adding noise).

\subsubsection{Simulation of an infarcted myocardium}

Since the purpose of the method presented here is to enable measuring Strain and SR at myocardial layers across the LV wall, the locality of the Strain calculations was tested by modifying the above model to simulate a condition of an infarct. At one segment, the longitudinal velocity gradient was set to zero for two layers (out of 4), as if that part of the muscle does not actively contract, but only dragged by other segments. Random Gaussian noise was added to the velocities (Noise $\sim \mathrm{N}(0, \sigma), \sigma=10 \%$ of maximal velocity in each direction.).

\subsection{Application to clinical data}

The method was applied in two clinical studies, to test its capability to quantitatively analyze the LV regional function. In the first study, the results of the analysis were compared with diagnosis made by an expert echocardiographer, and in the second with angiographic findings. The two studies included patients who were admitted to St. VinzenzHospital for acute myocardial infarction (AMI), defined according to the Guidelines of the European Society of Cardiology. The studies were performed according to the regulations of the local ethical committee, which approved each study design. All patients gave informed consent for further evaluation of the anonymous results of their echocardiographic and heart catheterization examinations. 

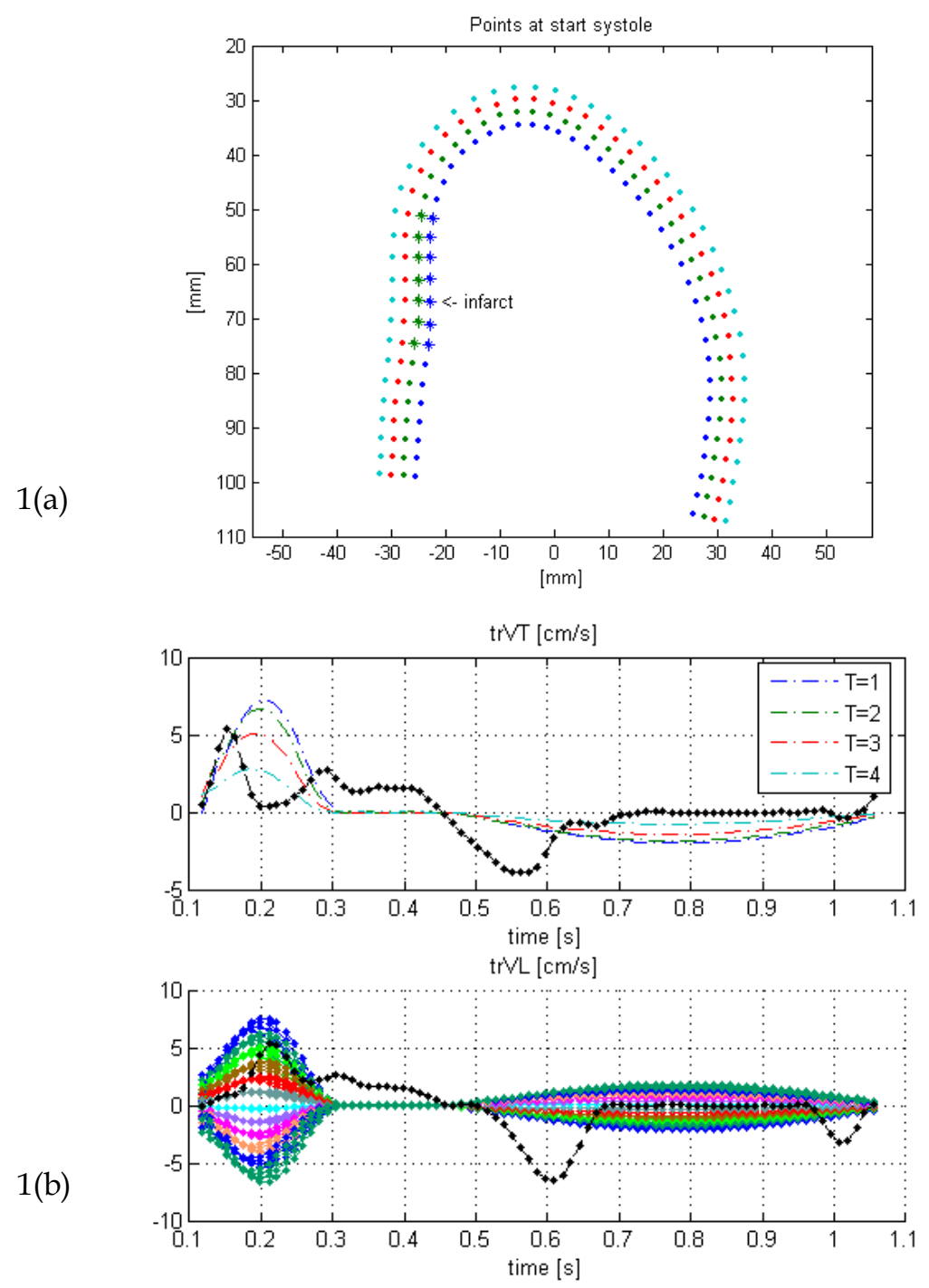

Fig. 1. (a): A model used for simulation of a non-transmural, regional infarct. A set of TP's (marked by ' ${ }^{\prime \prime}$ ), located at the inner 2 layers of the second segment, does not actively contract. The results of the analysis of the data generated by this model are displayed in Fig. 2. Fig. 1(b): Simulated velocities [cm/sec] of the TP's of Fig. 1(a) plotted as a function of time. Upper subplot - transmural velocities at the 4 lines of points (layers) from the endocardium $(\mathrm{T}=1)$ to the epicardium $(\mathrm{T}=4)$. Bottom subplot -56 colored curves (colors used just to differentiate among the curves) depicting longitudinal velocities, as the function of time, at 56 TP's along the LV long axis (from the basal-septal region, to the apex and then to the basal-freewall). The dotted black lines denote example of genuine myocardial velocities. To these velocities, random Gaussian noise was added. Noise $\sim \mathrm{N}(0, \sigma), \mathrm{\sigma}=10 \%$ of maximal velocity in each direction. 


\subsubsection{Comparison with an expert echocardiographer}

Echocardiographic examination usually includes qualitative assessment of wall motion, by inspection of the changes of length and thickening of each myocardial segment, where each segment comprises the whole width of the myocardial wall (Cerqueira et al., 2002). This process is usually denoted as 'regional analysis' and is applied by sectioning the LV myocardium to 18 myocardial segments, as proposed by the American Society of Echocardiography (Cerqueira, et al., 2002).

The patient group consisted of 9 patients (mean age $63 \pm 14$ years), who had symptoms occur within twelve hours of admission, and all were immediately transferred to the catheterization laboratory for coronary angiography and percutaneous coronary intervention. Based on the results of coronary angiography, 1 was diagnosed as having no coronary obstruction, and 8 diagnosed as suffering from stenosis in one of their main coronary arteries. Standard echocardiographic examinations were performed within 48 hours of admission, following the in-hospital routine patient management. For each subject -3 apical views were acquired (2 Chamber, 4 Chamber and Apical long-axis (Aplax) views), yielding a total of 162 segments. For each view and each myocardial segment, a score was given by an expert Echocardiographer studying the B-mode grey scale cine-loops. The score given: 1 - segment with normal kinematics; 2 - hypo-kinetic segment; 3 - a-kinetic segment; 4 - diskinetic segment.

The study focused on the systolic function, using two common measures - peak systolic Strain and peak systolic SR. These measures were compared to the score given by the expert. Longitudinal and transmural Strain and SR were calculated for each TP as explained above, and for each segment by averaging for all TP's of this segment. Peak systolic strain values were determined for each segment by finding during systole the minimal strain value in the longitudinal direction (contraction), and maximal strain values in the transmural direction (thickening).

\subsubsection{Statistical analysis}

The analysis of the results for all $(n=9)$ subjects above included calculation of the significance of the separation of the (162) segments into the four kinetic groups, according to the two motion parameters. Analysis of variance (ANOVA) was used to estimate the difference between the four groups. When the ANOVA test resulted in positive significance, post hoc tests (Scheffe) were used. The analysis was performed for the results of the smoothing performed by the wavelets method and by the polynomial fitting method.

\subsubsection{Comparison with angiographic results - Qualitative study}

A different patient group consisted of 21 patients (mean age $64 \pm 12$ years), selected following emergency admission for AMI. They had symptoms occur within twelve hours of admission, and all were immediately transferred to the catheterization laboratory for coronary angiography and percutaneous coronary intervention. Based on the results of coronary angiography, 5 were diagnosed as having no coronary obstruction and 16 
diagnosed as patients who suffer from stenosis in one of their main coronary arteries. Standard echocardiographic examinations were performed within 48 hours of admission, following the in-hospital routine patient management.

For each subject, three apical views were acquired (2ch, 4ch and Aplax) as well as short axis views at the basal-papillary level. The B-mode cine-loops were processed as described above, the data velocity data was smoothed as above, and the Strain and SR parameters were derived as described above. The evaluation was done by producing M-mode maps that help visualize each parameter as a function of time and the longitudinal location along the septal base - the apex - the lateral base. Such maps were produced for each of the above parameters, for each of the three myocardial layers. The M-mode maps, especially the Strain and SR maps, allow straightforward recognition of regions of reduced contractibility. These regions were visually compared with the areas of perfusion of the corresponding narrowed coronary artery, where the areas of perfusion of the main coronary arteries were divided according to the standard division of LV into 16 segments (Cerqueira et al., 2002). These results were also compared with the 'AFI' method, which performs over-smoothing of the data and does not provide resolution across the LV wall.

\section{Results}

\subsection{Simulated data - A normal myocardium}

The results obtained when using the polynomial curve fitting method yielded errors calculated to be SSE $=549.6$, while the wavelet shrinkage (with the best choice of parameters) method yielded an error 2/3 smaller (383.2 in terms of SSE).

\subsection{Simulated data - An infarcted myocardium}

Fig. 2 shows the longitudinal velocities after de-noising/smoothing, as performed by either the wavelet shrinkage method or the polynomial curve fitting method. Each subplot in the figure depicts the longitudinal velocities at one of the four myocardial layers (left endocardium, the 2 at the center - midwall, right- epicardium), as the function of time during the cardiac cycle. In each subplot, the vertical axis depicts the location along the anatomical M-mode line, starting from the septal base, through the apex and ending at the lateral base. The first row of four subplots presents the results of the wavelet denoising method, the middle row presents the results of the polynomial curve fitting method and the bottom row presents the original simulated velocities before the addition of noise. The arrows in the subplots of the 2nd column, point at the segment of infarction at the subendocardial-midwall layers, while the arrows in the subplots of the 3rd column point at the adjacent normal segment at the midwall-epicardial layers. The original simulated velocities (bottom row) display the difference between the normal and infarcted layers: the normal segment has a smooth longitudinal gradient of velocities, which does not exist in the infarcted layers. As can be seen from this figure, the difference of the velocities distribution between the two inner layers and the two outer layers is still apparent after the smoothing operation performed by either the wavelet method or the polynomial curve fitting method. 

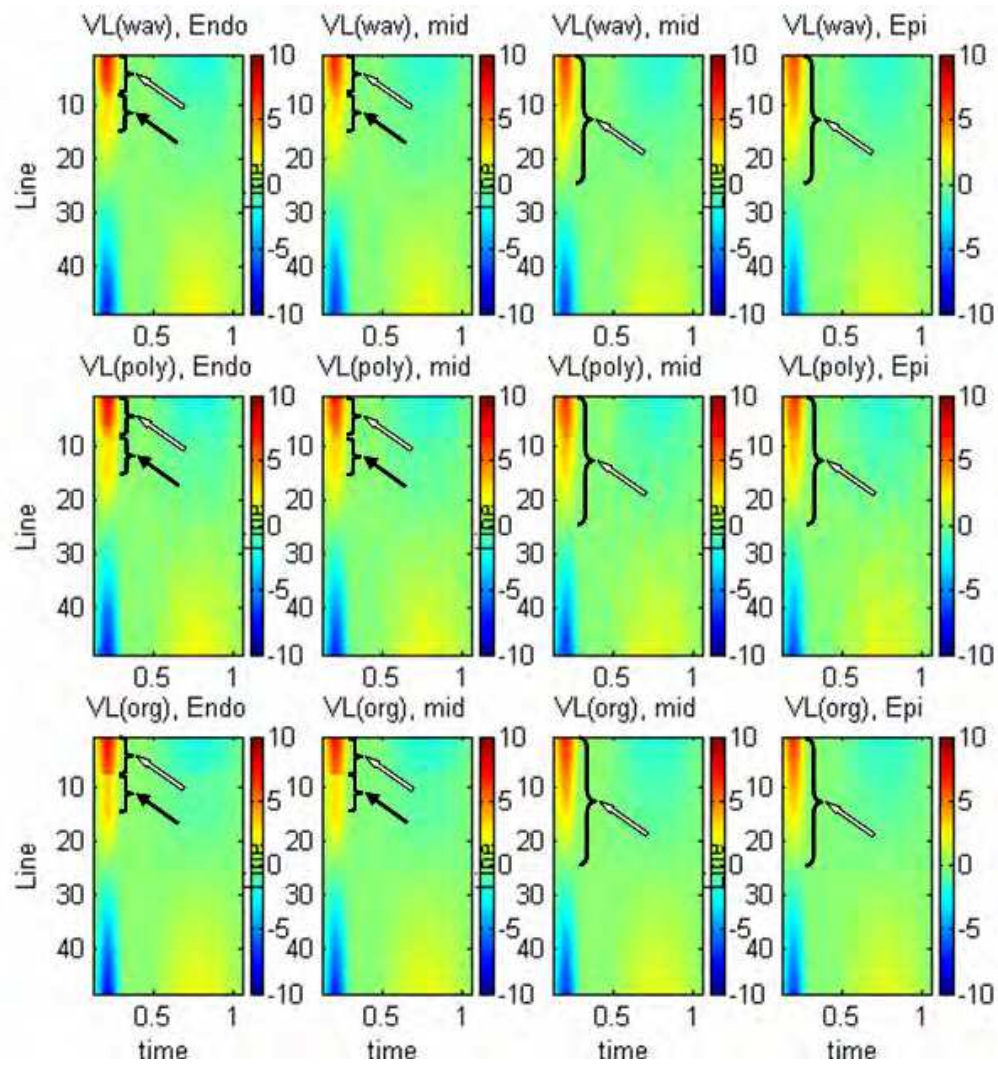

Fig. 2. Comparison of the de-noised longitudinal velocities, as performed by the wavelet vs. the polynomial fitting methods. Lower row - the original velocity distribution; Middle row - de-noising by polynomial fitting; Upper row - de-noising by wavelets. Each subplot is an M-mode plot of longitudinal velocities of the TP's (i.e. the ordinate depicts their longitudinal location) along the cardiac cycle, i.e. as a function of time (Abscissa). The black arrows emphasize the 'infarcted' segment (2 left columns), while the white arrows point to the 'normal' contracting segments ( 2 upper segments in the 2 left columns, and the full segments at the 2 right columns).

\subsection{Clinical data - Comparison with diagnosis performed by an expert echocardiographer}

The qualitative 'regional analysis' of wall motion, that was performed by the echocardiographer for the 9 patients included in this study, yielded quantification of function of 162 segments: 83 were diagnosed as normal and 79 with different degrees of a-kinetics.

In Fig. 3, two examples are given of color mapping of peak systolic longitudinal and transversal strain values. In Fig. 3(a) there are strain maps of a healthy subject, and Fig. 3(b) there are strain maps of a patient with a large LAD AMI (as validated also by angiography). The color at each segment denotes the value of the peak systolic strain according to the color map on the right side of the figure. The color map chosen is such that enables a clear 
distinction between the contracting segments (red colors) in the longitudinal direction (left panels) and segments that are stretched by the neighboring segments (blue color). In the transversal direction the normal segments appear in blue, as they become thicker during systole. Visual comparison of the results for the two subjects illustrates that for the normal subject the peak systolic strain values are homogenous along the myocardium and that for the pathological case a region near the apex appears that does not contract at all.

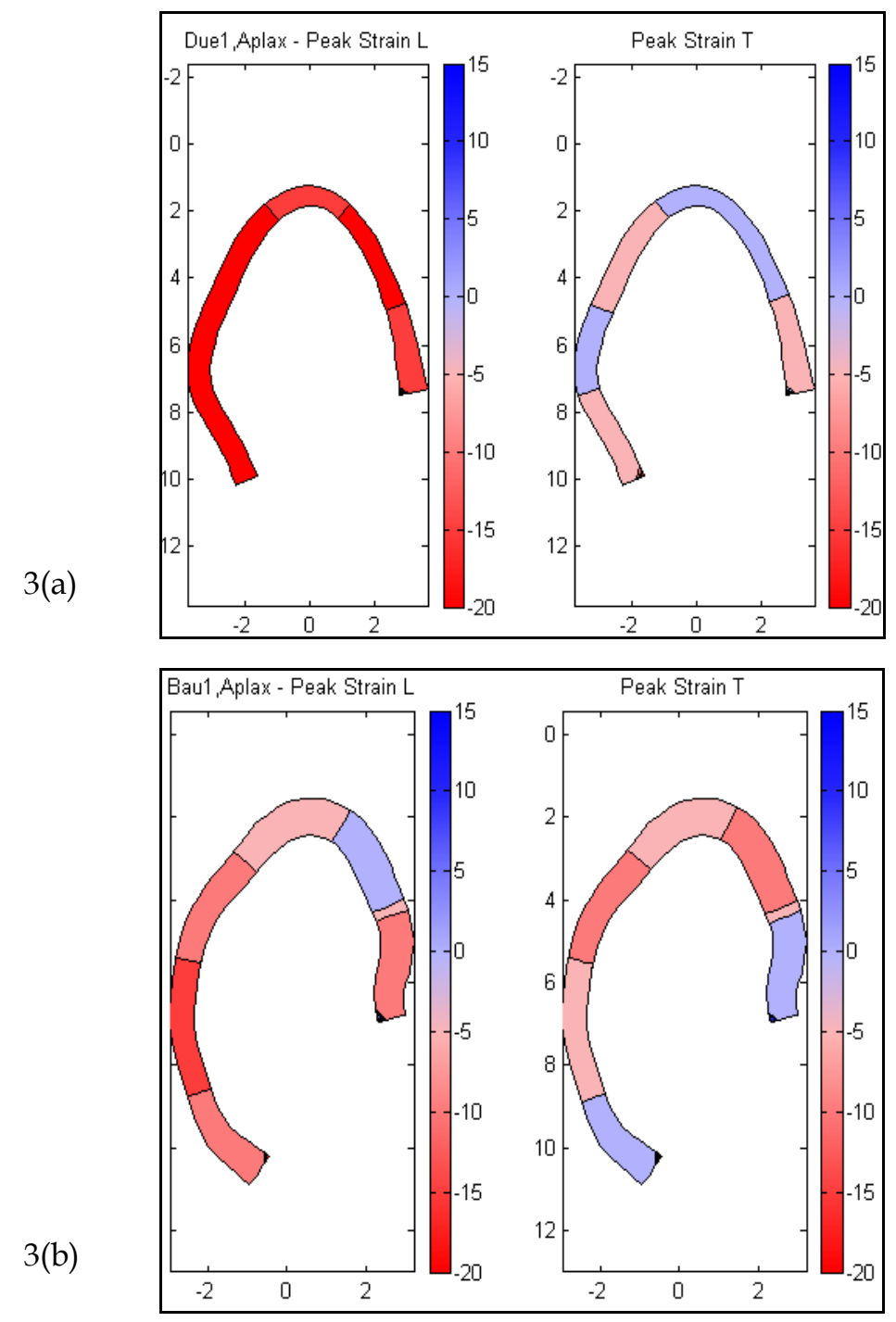

Fig. 3. Peak systolic strain values for each segment, calculated from data obtained from a normal subject (a) and from a patient with an infarct at the LAD (b). For each case: Left- peak systolic longitudinal strain (marked as 'Peak Strain L'), right - peak systolic transmural strain (marked as 'Peak Strain T'). 
Similarly, the results of the analysis of the peak systolic SR values at each segment, for the same two subjects, are depicted in Fig. 4. In Fig. 4(a) the results for a healthy subject are depicted, and in Fig. 4(b) for a patient with a large LAD AMI. The difference between the normal peak systolic SR maps and the pathological ones is clear.
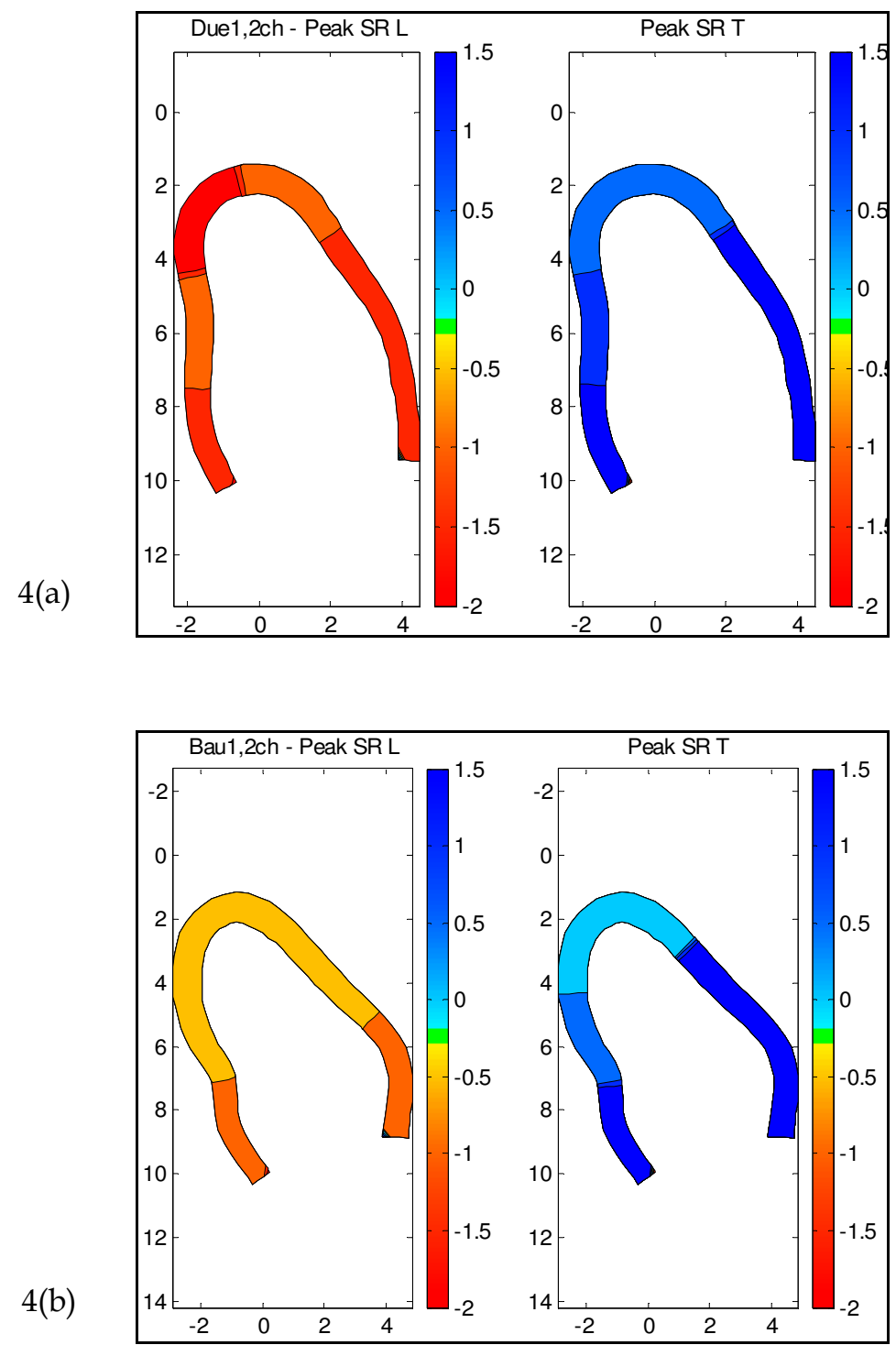

Fig. 4. Peak systolic SR values for each segment, calculated from data obtained from a normal subject (a) and from a patient with an infarct at the LAD (b). For each case: Left peak systolic longitudinal SR (marked as 'Peak SR L'), right - peak systolic transmural SR (marked as 'Peak SR T'). 
The statistical analysis of the results for all (9) subjects included calculation of the significance of the separation of the (162) segments into the four kinetic groups, according to the two motion parameters. The ANOVA analysis for the results of the smoothing performed by the wavelets method demonstrated that the peak systolic Strain results yielded a significant difference between the four groups: for the longitudinal peak Strain parameter, Slmax, $\mathrm{p}<0.001$, and for the transversal peak Strain parameter, Stmax, $\mathrm{p}<0.001$. The Scheffe test for Slmax showed significant differences between all groups except the diskinetic and the a-kinetic groups. The Scheffe test for Stmax found significant differences only between the normal group and the three pathological groups (Fig. 5, subplots (a) and (b)). Similarly, the ANOVA analysis of the peak systolic SR results yielded a significant difference between the four groups for the longitudinal peak SR parameter, SRImax, $\mathrm{p}<0.001$, and for the transversal peak SR parameter, SRtmax, $\mathrm{p}<0.001$. The Scheffe test for SRlmax showed significant differences between all groups except the dis-kinetic and the akinetic groups. The Scheffe test for SRtmax found significant differences only between the normal group and the diskinetic and a-kinetic groups (Fig. 5, subplots (c) and (d)).

When comparing these results to the ANOVA analysis for the results of the polynomial smoothing - it is evident that for both the longitudinal peak Strain parameter, Slmax, and the longitudinal peak SR parameter, SRlmax, $\mathrm{p}<0.001$, only the normal group can be separated from the 3 pathological groups (the hypo-kinetic, the diskinetic and a-kinetic groups) - Fig. 5, subplots (e) and (g), as well as (f) and (h). It is also evident that the entire peak systolic values are smaller (and the range narrower) when the smoothing is performed by the polynomial fitting (Abscissas of Fig. 5, subplots (e) to (h)) versus the wavelets smoothing (Abscissas of Fig. 5, subplots (a) to (d)).

\subsection{Clinical data - Comparison with angiographic results - Qualitative study}

This patient group consisted of 21 patients, of whom 5 were diagnosed by angiography as having no coronary obstruction and 16 diagnosed as patients who suffer from stenosis in one of their main coronary arteries. The standard echocardiographic examinations were post processed to produce the Strain and SR maps, as follows:

\subsubsection{Healthy subjects - Homogenous strain and SR along the myocardium}

M-mode maps of longitudinal SR and of longitudinal Strain are presented for two healthy subjects (Fig. 6) (b),(d). The upper row of maps depicts the results for the endocardial layer, the second from top row - midwall layer, the 3rd row from top - epicardial layer, the 4th row from top - the results of the 'AFI' method, which over-smoothes the data. In each map, the vertical axis represents the location along the anatomical M-mode line, starting from the septal base, through the apex and ending at the lateral base, while the horizontal axis depicts time. As can be seen in these maps, the pattern is homogenous along the myocardium (i.e. along the vertical axis). The red colors represent shortening, the blue colors stretching of the muscle.

\subsubsection{Healthy subject - Normal strain in short axis views}

The short axis views of healthy subjects demonstrated that circumferential strain is more significant at the endocardial layer than at the epicardial layer (Fig. 7). These differences were expected from geometric considerations and the assumption of tissue incompressibility, because concentric shells of myocardium must undergo proportionally greater changes in dimension for shells of smaller radius. The three subplots of Fig. 7 depict 


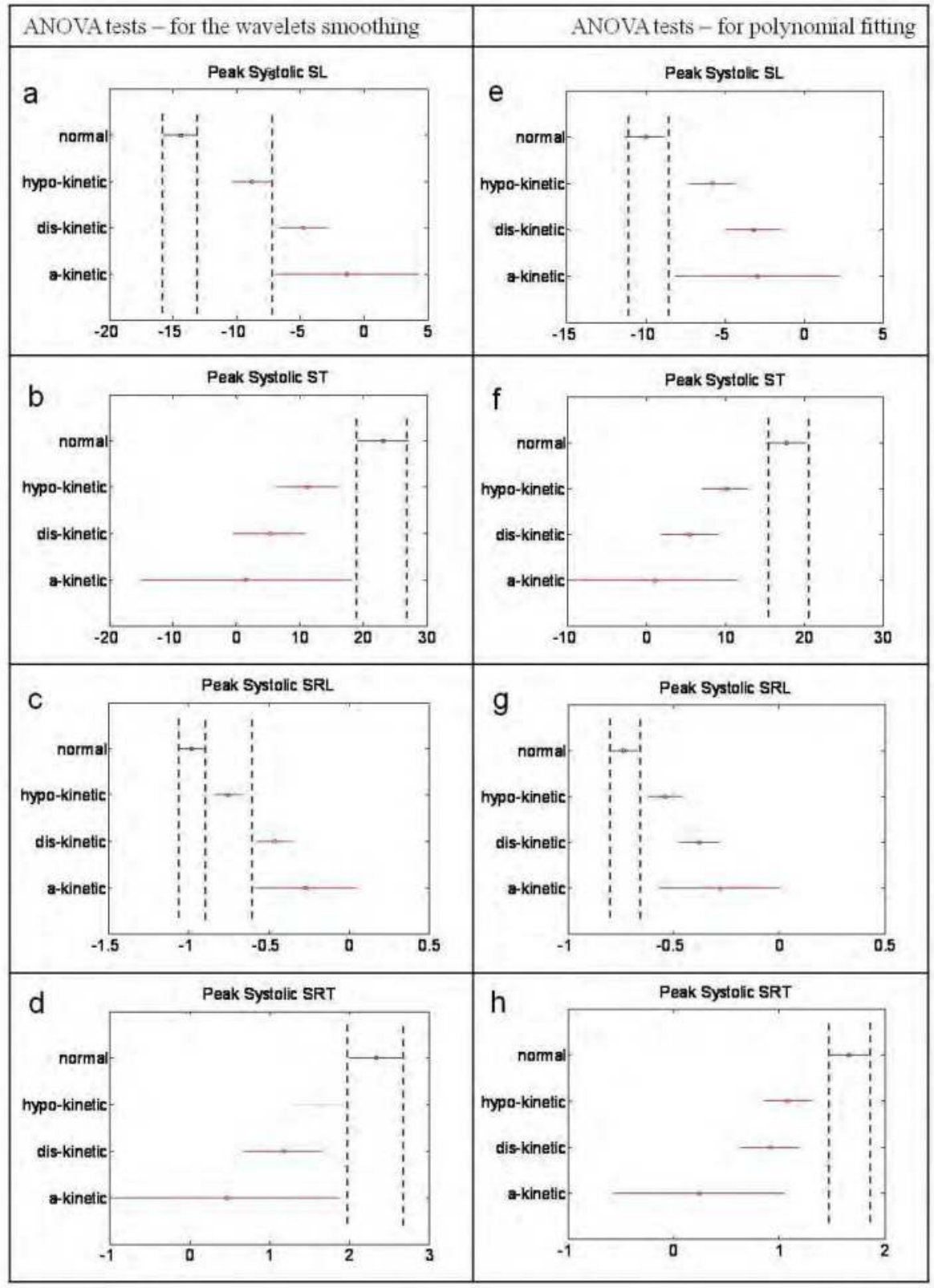

Fig. 5. Scheffe results for peak systolic longitudinal Strain (a, e), peak systolic transmural Strain (b, f), peak systolic longitudinal SR (c, g), peak systolic transmural SR (d, h). 162 segments are included in the analysis, 83 with normal contraction, 79 with different degrees of pathologies (45 segments hypo-kinetic; 29 diskinetic; 5 a-kinetic). Figures 5 (a-d) are results after wavelet smoothing, and figures $5(\mathrm{e}-\mathrm{h})$ are results after polynomial smoothing. 

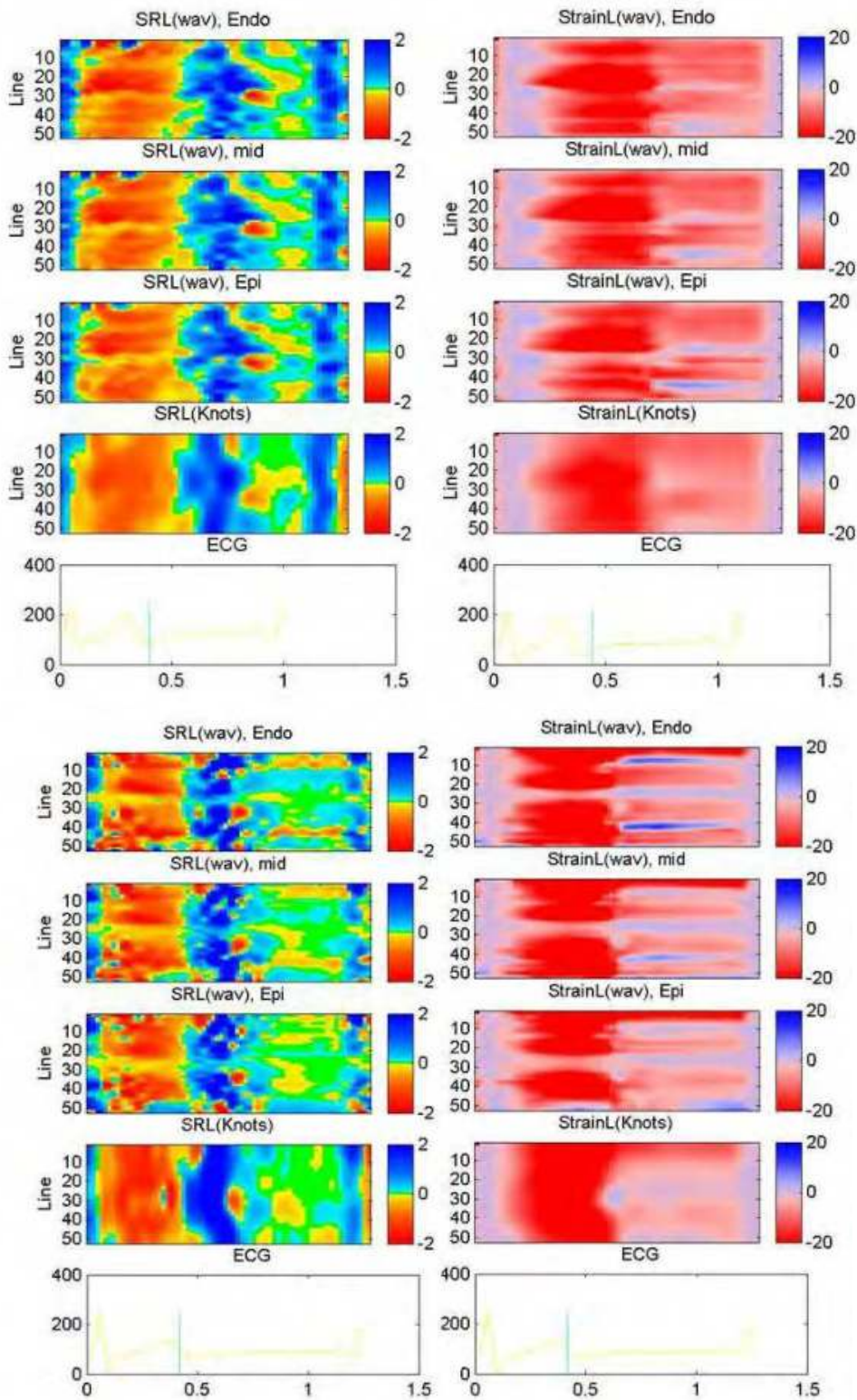

Fig. 6. Color-coded maps of results for 2 normal subjects that compare the wavelet analysis, which separates the 3 myocardial layers (1st row - subendocardium; 2nd row - midwall; 3rd row - epicardium), to the analysis that averages over the wall thickness (4th row). Columns 1 and 3: The longitudinal strain rate (SRL). Columns 2 and 4: longitudinal strain (StrainL). Each subplot is an M-mode plot (i.e. the ordinate depicts the longitudinal location), as a function of time (Abscissa - along the cardiac cycle). 
3 presentations, at 3 different locations (different sets of TP's, at locations 14, 30, 38), of the Strain traces for a healthy subject. In each subplot, the graphs on the left show the circumferential and radial Strains, at the 4 TP's marked by crosses at the graph on the right. It can be clearly seen that in all locations the amplitude of the circumferential strain is larger at the sub-endocardium than at the sub-epicardium.
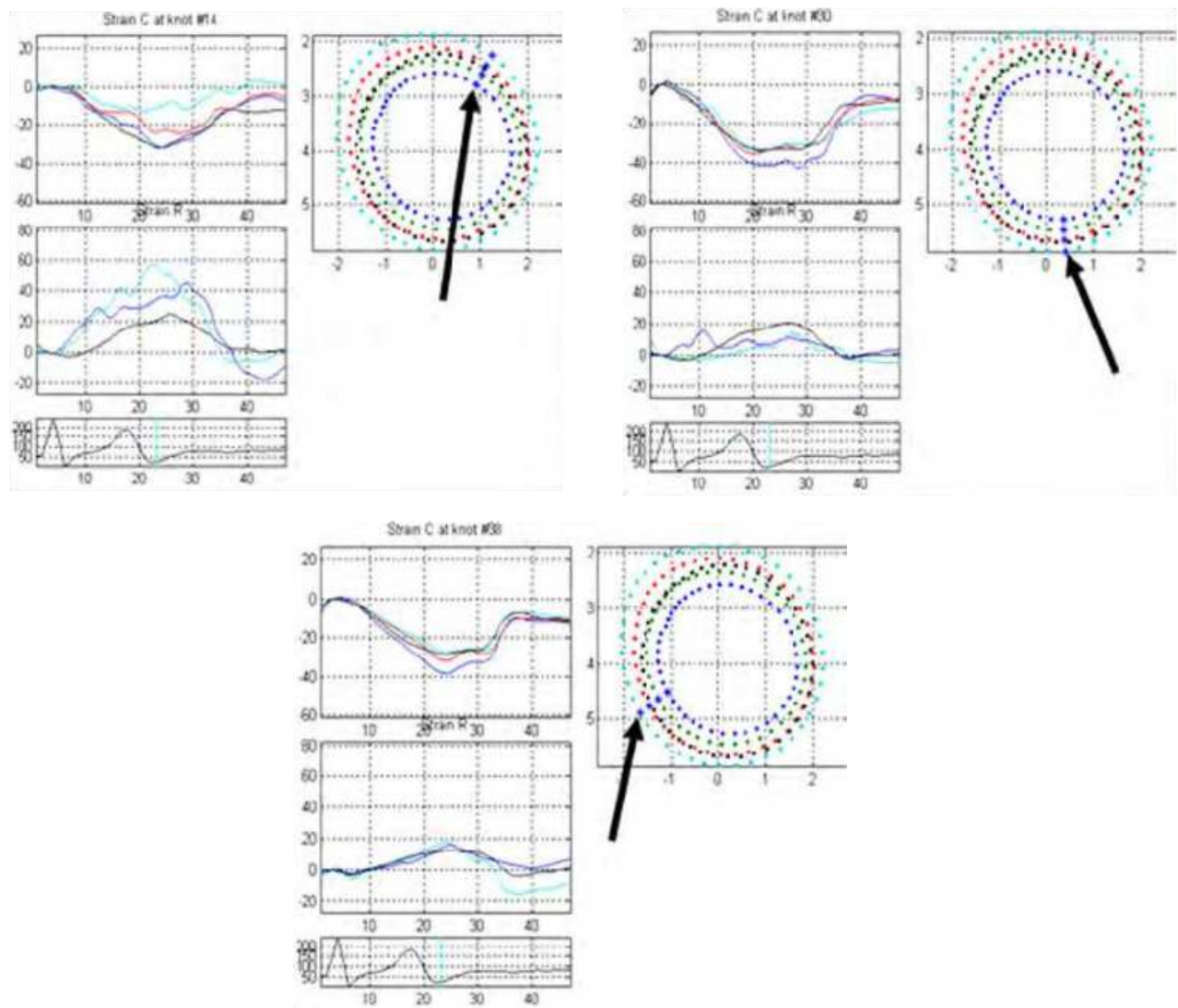

Fig. 7. Three examples of circumferential and Radial Strains, as measure from short-axis echocardiograms from a normal subject. In each example, the right subplot shows the 4 lines of $\mathrm{TP}^{\prime} \mathrm{s}$, with 4 specific points (marked by the arrow) that their time-dependant strains are depicted: The upper left subplot in each example depicts the circumferential strains, while the lower subplot depicts the radial strains.

\subsubsection{Post-AMI patients - Strain/SR results vs. angiography findings}

Out of the total of 21 subjects studied, 16 were post-AMI patients diagnosed with pathologies limited to a specific region. The detection of the location of each AMI, accomplished by using the M-mode Strain and SR maps, was validated by comparing the relevant segments with the segments predicted to be affected by the stenosis of the appropriate coronary artery, according to the AHA standard perfusion segmentation maps (Cerqueira et al., 2002). 
Two examples are given here. Fig. 8 displays the results of the analysis for a patient with LAD stenosis, and Fig. 9 for a LCx stenosis patient. The left column in each figure is a set of M-mode maps of longitudinal strain, while the middle column is a set of maps of the transmural strain, where the upper row relates to the endocardial layer, the second from top relates to the midwall, the third from top relates to the epicardial layer, and the bottom row relates to the over-smoothed strain, calculated by the 'AFI' method. The region of reduced contractility is marked by the black arrow in the maps and by the red area superimposed on the B-mode gray scale image. Superimposed on this same B-mode image of the LV long axis are also the line of 'knots' (large color dots), calculated from the 4 lines of TP's by the 'AFI' method, as well as the Region of Interest in which the tracking and the analysis were performed.
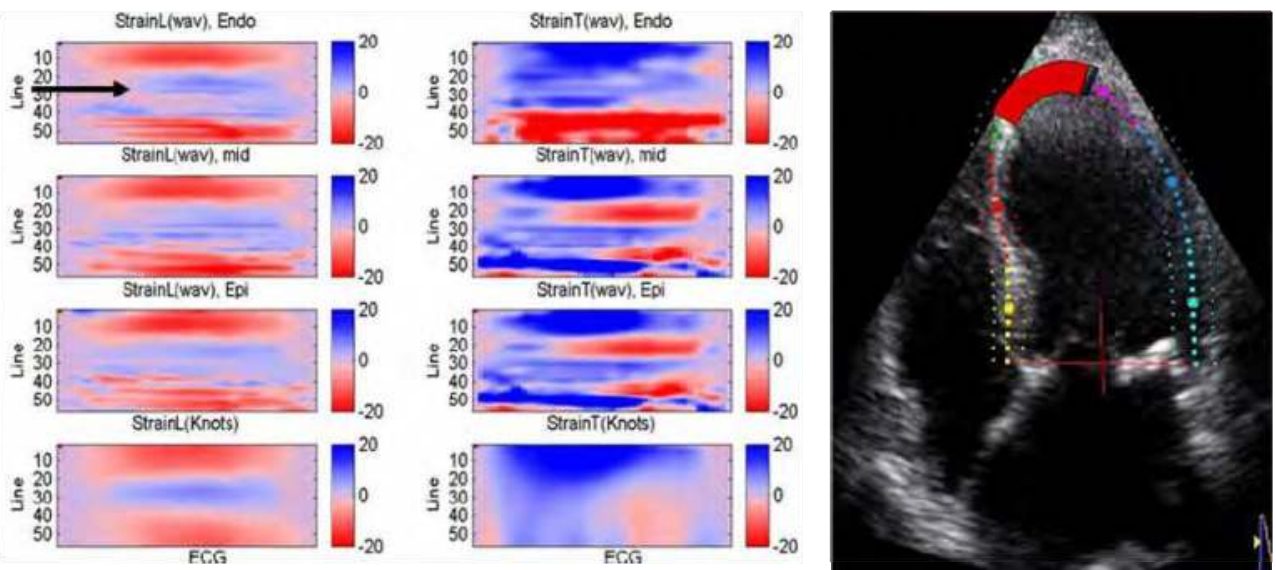

Fig. 8. Example of Strain results, as filtered by the wavelet method, calculated from long-axis echocardiogram of a patient with an AMI at the LAD. The left column subplots depict the longitudinal Strain, while the right column subplots depict the transverse Strain. Each subplot is an M-mode plot of Strain at different locations along the cross-section (i.e. the ordinate depicts the longitudinal location), along the cardiac cycle, i.e. as a function of time (Abscissa). The numbers (1-56) along the Ordinate reflect the TP number, as seen superimposed on the B-mode frame on the right hand-side, where point 1 is at the basalseptal location (lower-left), while point 56 is at the basal-freewall (lower right). Upper row Strain at the subendocardium; 2nd row-Strain at the midwall; 3rd row - Strain at the epicardium; 4th row - Strain as calculated when values are first average across the wall (the AFI method). The black arrows on the left emphasize the infarcted region, also marked in red on the B-mode image on the right. 

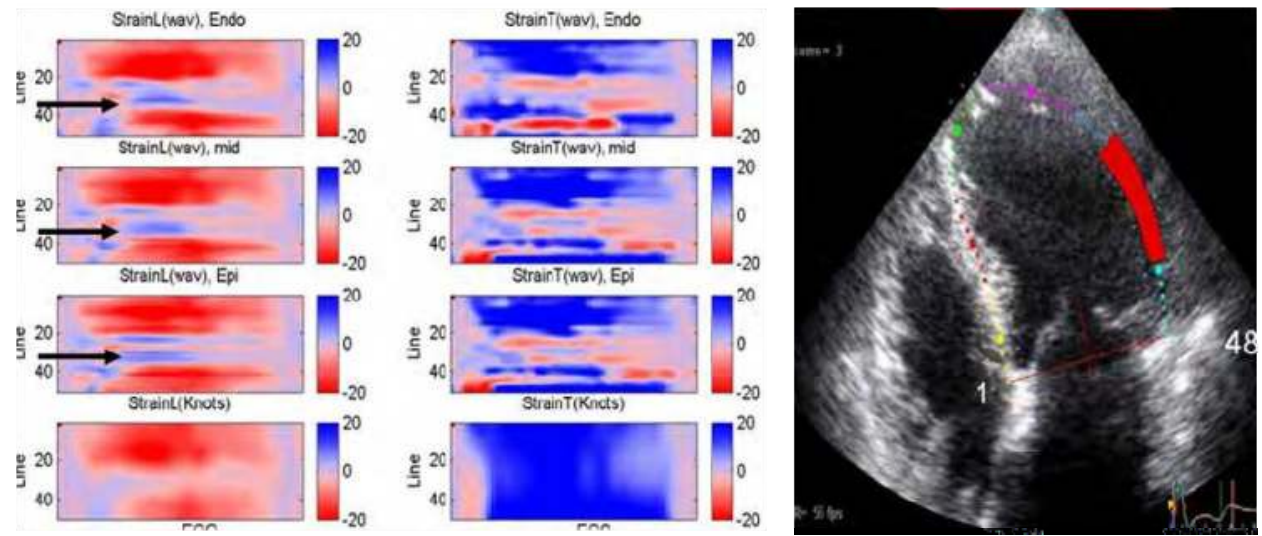

Fig. 9. Example of Strain results, as filtered by the wavelet method, calculated from long-axis echocardiogram of a patient with an AMI at the LCX. Details are similar to those given for Fig. 10 (except that here only $48 \mathrm{TP}^{\prime} \mathrm{s}$ have been assigned).

The total data set comprised of 63 sets ( 3 views acquired from 21 subjects: 5 healthy subjects, 2 patients suffering from RCA occlusion, 3 LCx and 11 LAD), which were graded according to the level of agreement with the angiography findings: matching of the coronary artery location - 1; partial matching - 2; no match - 3. Out of the 63 sets, 41 (65\%) showed a good agreement, 19 (30\%) partial matching, while in $3(5 \%)$ there was no match at all.

Most of the cases of partial (or no) match between the diagnosis based on the Strain and SR maps and the diagnosis based on the angiography findings, were cases that were categorized as normal, and the Strain and SR maps showed a region with reduced contraction. This outcome may be either the result of low quality tracking in these regions (especially near the apex), or of true pathology, since all subjects were symptomatic and underwent an angiographic study, and were diagnosed according to its findings.

\subsubsection{Ability to diagnose small AMls}

Since the method presented here produces maps that reveal motion and contraction at each of the three myocardial layers separately, it is now possible to detect smaller and nontransmural AMIs. Fig. 10 shows an example of a patient with a small LAD AMI, where its location is marked by a black arrow. The depressed contraction is mostly seen at the endocardial layer, while at the epicardial layer some contraction exists in nearly all regions. It can also be seen that while the depressed contraction caused by the occluded LAD artery is visible in the Strain maps produced by the new method, the maps produced by the 'AFI' method do not have the sufficient resolution to reveal the depressed Strain of the ischemic or infarcted region (bottom row). 

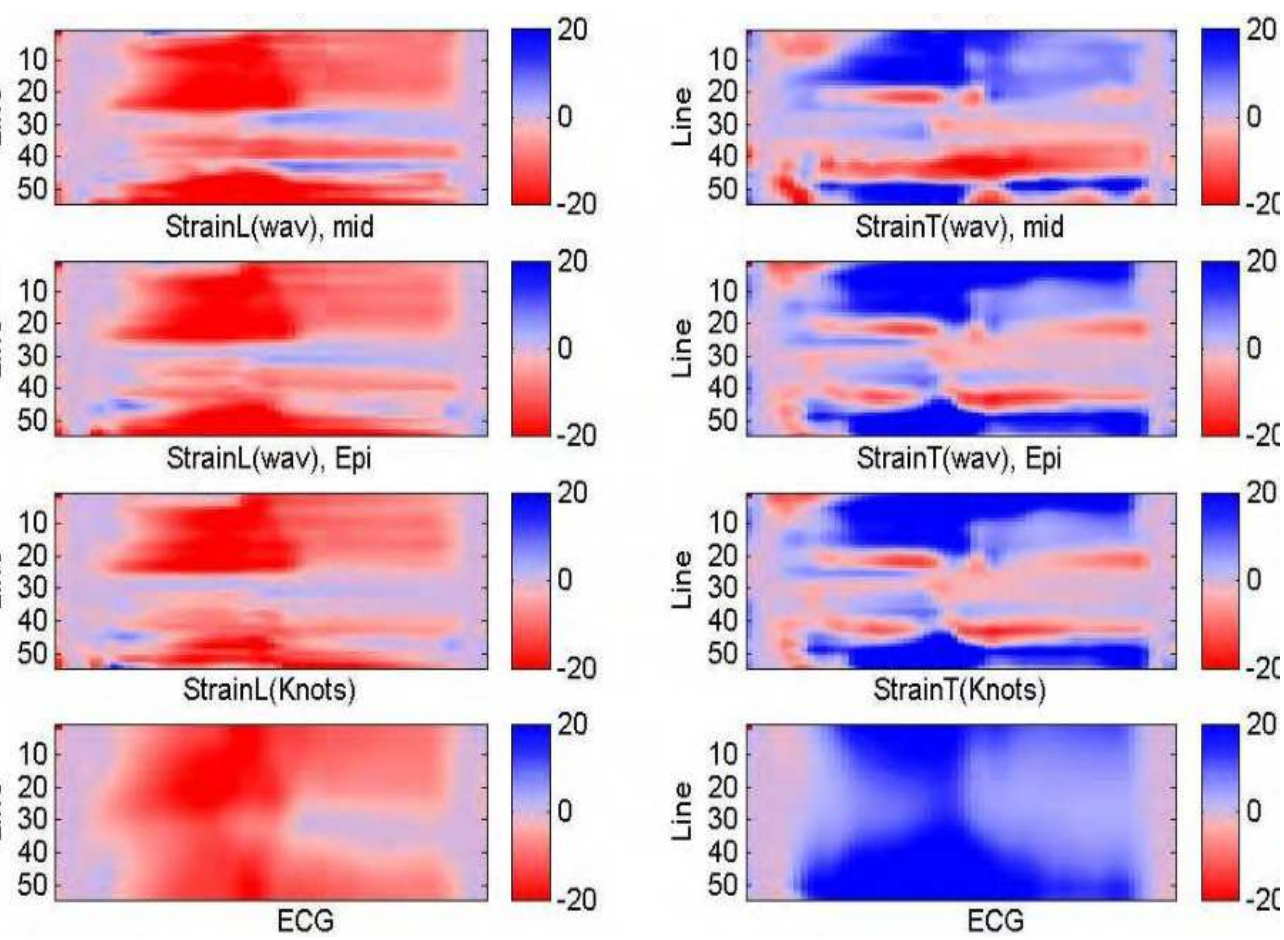

Fig. 10. Example of Strain results, as filtered by the wavelet method, calculated from longaxis echocardiogram of a patient with a small AMI at the LAD. Details are similar to those given for Fig. 10. This example demonstrates that the M-mode plot of Strain, as calculated by the AFI method (the lower row) - seems normal. (here $56 \mathrm{TP}^{\prime}$ s have been assigned).

\section{Discussion}

The new method presented here, based on analysis of echocardiographic B-mode sequences, allows computing myocardial motion and contraction properties in all segments of the 2D myocardial cross-section, thus overcoming the intrinsic limitations of the clinically accepted Doppler-based techniques. Clinical experience confirms that the motion of even a normal heart is rarely along the direction of the myocardial wall, thus accurate results of Dopplerbased techniques are obtained perhaps at the septum. Additionally, the Doppler-based techniques obviously do not provide both longitudinal and transverse measurements. They also cause reduction of the frame rate of the B-mode image acquisition, since the two measurements cannot be performed simultaneously. Therefore, Doppler data is usually acquired on a small set of image lines, (e.g. 16 samples in GE's Vivid3 and Vivid7), which increases the inaccuracy of the measurement.

The recently presented 'AFI' method (Rappaport et al. 2006; Leitman et al. 2004; Reisner et al. 2004) provides functional and clinically useful information throughout the imaged myocardial cross-section. This method, though, employs robust smoothing techniques and therefore the spatial resolution is relatively low. Moreover, since the motion information is 
represented for only one line of points along the longitudinal axis of the myocardium, variations between myocardial layers cannot be detected.

The accuracy of the high resolution method presented here, of measuring regional 2D strain, suffers from the same 2 inherent shortcomings as its earlier version (Reisner et al. 2004; Rappaport et al. 2006): it depends on tracking quality, and on the inability to follow the gross heart movement with the probe. The second shortcoming is specifically troublesome when $2 \mathrm{D}$ data is acquired by ultrasound 2D probe, held in place, while the motion of the heart is three-dimensional, causing acquisition of practically a different cross-section of tissue at each different instant. This causes reduction of the correlation of the speckle pattern in the analyzed 2-D sequences, and produces weaker relations between frames. Similar effects are observed for out-of-plane motion, which may cause intra-cardiac structures (for example, papillary muscles) to penetrate and exit the imaged plane, a limitation that is shared by other 2-D modalities.

Additional shortcoming is related to the calculation of SR, which is performed by spatial derivation of the velocities. Thus the error inherent in the velocity data is magnified. The other methods, however, also share this shortcoming.

While the above shortcomings are shared by all other ultrasound methods, the method presented here takes advantage of the basic data-set, the velocities at a set ( 4 by (50 to 60) by (50-70) time instants) of TP's, to provide 2D Strain and SR tensors at each myocardial point. This was demonstrated to provide analysis of local deformations (orientations and magnitudes) at different myocardial layers, including their variations in time, along and across the myocardial wall.

The performance of the method, using the wavelet shrinkage method, was found to be slightly better in terms of the sum of square errors, and much better in terms of computational load. A model was also used to demonstrate the feasibility of the method to detect a region comprising of 2 layers that do not contract, simulating a 'regional nontransmural AMI'. Both smoothing methods allowed discrimination between the two 'normal' layers and the two 'infarcted' layers at this region.

The preliminary clinical studies compared the diagnosis performed by the echocardiographer for the 162 segments with the set of parameters calculated during systole - the peak systolic Strain, and the peak systolic SR. The results obtained using the calculations of the longitudinal Strain and longitudinal SR have been found to be in good agreement with the expert's diagnosis, better than the agreement found for the transmural calculations. This result was expected since the width of the myocardium muscle is relatively small (only 4 TPs), and so the reliability of the calculations in the transmural direction is lower.

The comparison of the angiographic results with the regional Strain and SR of 63 data sets (21 subjects, 3 apical views for each subject) analyzed by the 2 smoothing methods, demonstrated that the 2 smoothing methods provide better spatial resolution than the AFI method, specifically among the different myocardial layers across the wall. The wavelet denoising outperforms the polynomial fitting, as demonstrated in Fig. 5, allowing differentiation normal vs. pathological regions, but also between hypo-kinetic regions and diskinetic or a-kinetic regions. The separation into 3 groups was evident from the peak 
systolic Strain measurements, as well as from the peak systolic SR. The comparison to the angiographic study was done by observing the contraction patterns in M-mode maps of the Strain and SR of the four myocardial layers during the entire cardiac cycle. The results were also compared with the Strain and SR calculated by the 'AFI' method.

During systole, specifically during the ECG S-wave, the normal SR pattern reflects the myocardial shortening in the longitudinal direction and extension in the transversal direction. In most of the normal cases studied here, this contraction is apparent as a simultaneous shortening of all myocardial layers. In contrast, the contraction in the cases of the abnormal patients presented a different behavior: at the anomalous regions the myocardium did not contract at all in the longitudinal direction, or was even extended, while it shortened in the orthogonal direction. In some cases, diversity between the layers was observed, where some layers functioned normally and some abnormally. The normal SR pattern during the E wave was, as expected, of myocardial extension in the longitudinal direction and shortening in the transversal direction. In abnormal hearts, either the longitudinal extension did not occur or was weaker than the extension noticed in normal hearts.

A good agreement was found between the results of the present study, the results of the 'AFI' method and the areas supplied by the narrowed artery (as found by the angiography study). In those cases where there was no agreement or only partial agreement between the findings of the present study (based on the Strain - SR maps) and the angiography findings, the majority of the cases were those that were clinically categorized as normal, while the Strain - SR maps showed a region with reduced contraction. This outcome can be the result of either low quality of tracking in those regions (especially near the apex), or reduced functionality due to partial ischemia that the angiography failed to diagnose.

The examination of the circumferential strain of normal subjects (in short axis views) showed higher Strain values along the endocardial layer than the epicardial layer, similar to reported results (Helm et al. 2005), and as expected from geometrical considerations and tissue incompressibility. These results, therefore, demonstrate the reliability of the method of regional analysis.

Some of the smaller AMI's that were found by angiography and were also so diagnosed by the present method, but were not detected by the 'AFI' method. The method presented here has the potential to detect smaller AMI's and non-transmural AMI's better than currently available techniques, but this needs to be assessed in large-scale clinical studies.

The advantage of visualizing the Strain and SR as M-mode maps allows examining not only the peak Strain - SR, but also their temporal evolution. This visualization provides important information about the regional function of the left ventricle and yields semiquantitative assessment of wall motion. Another advantage of visualizing Strain and not just motion information is its reduced sensitivity to translation of the whole heart.

In conclusion, the method presented here provides region information regarding the myocardial distribution of Strain and SR, which allows functional differentiation among the myocardial layers. The 2 smoothing methods provide good results, with the wavelet denoising outperforming the polynomial fitting, allowing differentiating normal from pathological regions, but also between hypo-kinetic regions and diskinetic or a-kinetic 
regions. The higher spatial resolution may be useful in diagnosing transmural versus nontransmural AMI's, and due to the visualization methods used - dyssynchrony of activation and pathologies of Strain evolution, e.g. post systolic shortening.

\section{Acknowledgments}

This study was supported by the Magneton program, the Chief Scientist, Ministry of Industry and Trade, and by the Technion VPR Fund for the promotion of Research. The Authors are grateful for the generous support.

\section{References}

Behar, V., Adam, D., Lysyansky, P., Friedman, Z., The combined effect of nonlinear filtration and window size on the accuracy of tissue displacement estimation using detected echo signals. Ultrasonics 2004; 41:743-753.

Bertrand, M., Meunier, J., Doucet, M., Ferland, G., Ultrasonic biomechanical strain gauge based on speckle tracking, Proc. 1989 IEEE Ultrason. Symp.: 859 - 863.

Bachner-Hinenzon N Ertracht O, Lysiansky M, Binah O, Adam D. Layer-specific assessment of left ventricular function by utilizing wavelet de-noising: A validation study. Med Biol Eng Comput. 2011 Jan;49(1):3-13. Epub 2010 Jul 20.

Cerqueira, M.D., Weissman, N.J., Dilsizian, V., Jacobs, A.K., Kaul, S., Laskey, W.K., Pennell, D.J., Rumberger, J.A., Ryan, T., Verani, M.S., Standardized Myocardial Segmentation and Nomenclature for Tomographic Imaging of the Heart, Circulation, 2002; 105: 539-542.

De Boeck, B.W.L., Cramer, M-J.M., Loh, P., Doevendans, P.A.F.M., Two-Dimensional Strain Imaging to Assess the Origin and Extent of Ventricular Preexcitation Associated With an Accessory Bypass, Circulation 2006;113: 835-839.

Desco, M., Ledesma-Carbayo, M. J., et al., Assessment of normal and ischaemic myocardium by quantitative m-mode tissue Doppler imaging, Ultrasound in Med. \& Biol., 2002; 28 (5): 561-569.

Donoho, D.L., De-Noising By Soft-Thresholding, IEEE Trans. on Information Theory, 1995; 41 (3): 613-627.

Donoho, D.L., Johnstone, I.M., Ideal spatial adaptation by wavelet shrinkage, Biometrika, 1994; 84 (3): 425-455.

Heimdal, A., Stoyen, A., Torp, H., Skjxrpe, T., Real-Time Strain Rate Imaging of the Left Ventricle by Ultrasound, J Am Soc Echocardiogr, 1998; 11 (11):1013-1019.

Helm, R.H., Leclercq, C., Faris, O.P., Ozturk, C., McVeigh, E., Lardo, A.C., Kass, D.A., Cardiac Dyssynchrony Analysis Using Circumferential Versus Longitudinal Strain Implications for Assessing Cardiac Resynchronization, Circulation, 2005;111: 27602767.

Herbots, L.,Maes, F., D’hooge, J, Claus, P., Dymarkowski, S., Martens, P., Mortelmans, L., Bijnems, B., Bogaert, J., Rademakers, F.E., Sutherland, G.R., Quantifying Myocardial Deformation Throughout the Cardiac Cycle: A Comparison of Ultrasound Strain Rate, Grey-Scale M-Mode and Magnetic Resonance Imaging, Ultrasound in Med. \& Biol., 2004; 30 (5): 591-598. 
Ledesma-Carbayo, M..J., Kybic, J., Desco, M., Santos, A., Sühling, M., Hunziker, P., and Unser, M., Spatio-temporal nonrigid registration for ultrasound cardiac motion estimation, IEEE Trans. Med. Imag., 2005; 24, (9), 1113-1126.

Leitman M, Lysyansky P, Sidenko S, Shir V, Peleg E, Binenbaum M, Kaluski E, Krakover R, Vered Z Two-dimensional strain-a novel software for real-time quantitative echocardiographic assessment of myocardial function, J Am Soc Echocardiogr, 2004; 17 (10):1021-1029.

Liel-Cohen N, Tsadok Y, Beeri R, Lysyansky P, Agmon Y, Feinberg MS, Fehske W, Gilon D, Hay I, Kuperstein R, Leitman M, Deutsch L, Rosenmann D, Sagie A, Shimoni S, Vaturi M, Friedman Z, Blondheim DS. A New Tool for Automatic Assessment of Segmental Wall Motion, Based on Longitudinal 2D Strain: A Multicenter Study by the Israeli Echocardiography Research Group. Circ Cardiovasc Imaging. 3(1):47-53, 2010.

Mallat, S., A Wavelet Tour of Signal Processing, Academic Press,1998.

Moore, C.C., Lugo-Olivieri, C.H., McVeigh, E.R., Zerhouni, E.A.: Three-dimensional systolic strain patterns in the normal human left ventricle: characterization with tagged MR imaging, Radiology, 2000; 214 (2): 453-466.

Nesto, R.W., and Kowalchuk, G.J., The Ischemic cascade: Temporal sequence of hemodynamic, electrocardiographic and symptomatic expressions of ischemia, American Journal of Cardiology, 1987; 59: 23C-30C.

O'Donnell, M., Skovoroda, A. R. and Shapo. B. M., Measurement of arterial wall motion using Fourier based speckle tracking algorithms, Proc. 1991 IEEE Ultrason. Symp., 1101-1104.

O'Donnell, M. Skovoroda, A.R.; Shapo, B.M.; Emelianov, S.Y.; Internal displacement and strain imaging using ultrasonic speckle tracking, IEEE Trans. Ultrason. Ferroelect. Frequency Contr., 1994, 41 (3): 314 -325.

Rappaport, D., Adam, D., Lysyansky, P., Riesner, S., Assessment of Myocardial Regional Strain and Strain Rate by Tissue Tracking in B-Mode Echocardiograms, Ultrasound in Med. \& Biol., 2006; 32 (8): 1181-1192.

Reisner, S., Lysyansky, P., Agmon, Y., Mutlak, D., Lessick, J., Friedman, Z., Global longitudinal strain: A novel index of left ventricular systolic function. J Am Soc Echocardiogr, 2004;17: 630-633.

Suffoletto, M.S., Dohi, K., Cannesson, M., Saba, S., Gorcsan, J., Novel Speckle-Tracking Radial Strain From Routine Black-and-White Echocardiographic Images to Quantify Dyssynchrony and Predict Response to Cardiac Resynchronization Therapy, Circulation. 2006;113: 960-968.

Sutherland, G.R., Di Salvo, G., Claus, P., D'hooge, J., Bijnens, B., Strain and Strain Rate Imaging: A New Clinical Approach to Quantifying Regional Myocardial Function, J Am Soc Echocardiogr, 2004; 17 (7): 788-802.

Unser, M., A Review of Wavelets in Biomedical Applications, Proc. Of the IEEE, 1996; 84 (4): 626-638.

Voigt, J.U. and Flachskampf, F.A., Strain and Strain Rate Imaging - New and Clinically Relevant Echo Parameters of Regional Myocardial Function, Z. Kardiol, 2004; 93: 249-258.

Voigt JU, Nixdorff U, Bogdan R, Exner B, Schmiedehausen K, Platsch G, Kuwert T, Daniel WG, Flachskampf FA, Comparison of deformation imaging and velocity imaging 
for detecting regional inducible ischaemia during dobutamine stress echocardiography. Eur Heart J, 2004; 25 (17):1517-1525.

Weidemann, F., Dommke, C., Bijnens, B., Claus, P., D’hooge, J., Mertens, P., Verbeken, E., Maes, A., Van de Werf, F., De Scheerder, I., Sutherland, G.R., Defining the Transmurality of a Chronic Myocardial Infarction by Ultrasonic Strain-Rate Imaging, Circulation, 2003; 107: 883-888.

Yip, G., Abraham, T., Belohlavek, M., Khandheria, B., Clinical Applications of Strain Rate Imaging, J Am Soc Echocardiogr, 2003; 16 (12): 1334-1342.

Yip, G., Khandheria, B., Belohlavek, M., Pislaru, C., Seward, J., Bailey, K., Tajik, A.J., Pellikka, P., Abraham, T., Strain Echocardiography Tracks Dobutamine-Induced Decrease in Regional Myocardial Perfusion in Nonocclusive Coronary Stenosis, J Am Col Cardiol, 2004; 44:1664-1671.

Zwanenburg, J.J.M., Götte, M.J.W., Marcus, J.T., Kuijer, J.P.A., Knaapen, P., Heethaar, R.M., van Rossum, A.C., Propagation of Onset and Peak Time of Myocardial Shortening in Ischemic Versus Nonischemic Cardiomyopathy Assessment by Magnetic Resonance Imaging Myocardial Tagging, J Am Coll Cardiol 2005; 46: 2215-2222. 


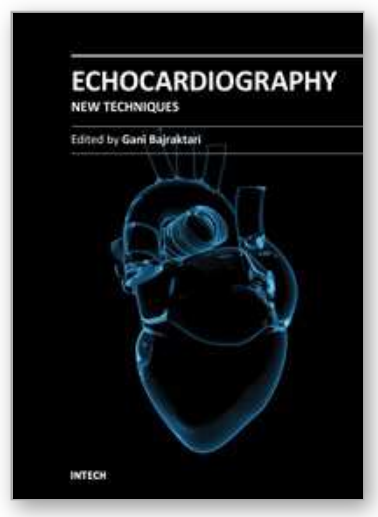

\author{
Echocardiography - New Techniques \\ Edited by Prof. Gani Bajraktari
}

ISBN 978-953-307-762-8

Hard cover, 218 pages

Publisher InTech

Published online 18, January, 2012

Published in print edition January, 2012

The book "Echocardiography - New Techniques" brings worldwide contributions from highly acclaimed clinical and imaging science investigators, and representatives from academic medical centers. Each chapter is designed and written to be accessible to those with a basic knowledge of echocardiography. Additionally, the chapters are meant to be stimulating and educational to the experts and investigators in the field of echocardiography. This book is aimed primarily at cardiology fellows on their basic echocardiography rotation, fellows in general internal medicine, radiology and emergency medicine, and experts in the arena of echocardiography. Over the last few decades, the rate of technological advancements has developed dramatically, resulting in new techniques and improved echocardiographic imaging. The authors of this book focused on presenting the most advanced techniques useful in today's research and in daily clinical practice. These advanced techniques are utilized in the detection of different cardiac pathologies in patients, in contributing to their clinical decision, as well as follow-up and outcome predictions. In addition to the advanced techniques covered, this book expounds upon several special pathologies with respect to the functions of echocardiography.

\title{
How to reference
}

In order to correctly reference this scholarly work, feel free to copy and paste the following:

Vitek Nili, Bachner-Hinenzon Noa, Lempel Meytal, Friedman Zvi, Reisner Shimon, Beyar Rafael and Adam Dan (2012). Assessment of Regional Longitudinal Stain by Using Speckle Tracking Echocardiography - A Validation Study, Echocardiography - New Techniques, Prof. Gani Bajraktari (Ed.), ISBN: 978-953-307-762-8, InTech, Available from: http://www.intechopen.com/books/echocardiography-new-techniques/assessment-ofregional-longitudinal-stain-by-using-speckle-tracking-echocardiography-a-validation-st

\section{INTECH}

open science | open minds

\author{
InTech Europe \\ University Campus STeP Ri \\ Slavka Krautzeka 83/A \\ 51000 Rijeka, Croatia \\ Phone: +385 (51) 770447 \\ Fax: +385 (51) 686166 \\ www.intechopen.com
}

\author{
InTech China \\ Unit 405, Office Block, Hotel Equatorial Shanghai \\ No.65, Yan An Road (West), Shanghai, 200040, China \\ 中国上海市延安西路65号上海国际贵都大饭店办公楼 405 单元 \\ Phone: +86-21-62489820 \\ Fax: +86-21-62489821
}


(C) 2012 The Author(s). Licensee IntechOpen. This is an open access article distributed under the terms of the Creative Commons Attribution 3.0 License, which permits unrestricted use, distribution, and reproduction in any medium, provided the original work is properly cited. 